\title{
Go Back To The Basics: Cardiac Surgery Trainees At The Time Of Covid-19.
}

\author{
Gabriella Ricciardi ${ }^{1}$, Raoul Biondi ${ }^{2}$, and Gabriele Tamagnini ${ }^{2}$ \\ ${ }^{1}$ Leiden Universitair Medisch Centrum \\ ${ }^{2}$ Villa Torri Hospital
}

May 11, 2020

\begin{abstract}
Keeping up with the surgical training might be difficult during the time of COVID-19 pandemic: with most of the health care resources dedicated to face this reality, trainees can improve themselves deep diving in scientific literature, study, Telemedicine and Social Media professional platforms. Moreover, they might be directly involved in COVID patient care, facing a still a still elusive disease with a high lethality rate. Often the frustration of having no valid treatment and a poor incisiveness on the natural course of the COVID19 could lead to a blue mood or a burnout. Eventually, the natural adaptability and the survival instinct prevail and teach us the real meaning of resilience. Every trainee has to be prepared for the second phase, when the new normality will force everyone to cohabit with the virus. Even the obvious teething troubles, this could be the right moment for all the Residents to "grow-up" and develop their own future Character.
\end{abstract}

\section{Central Message}

Even in time of forced step back in their personal ambitions and surgical practice, Residents can achieve self-improvement and work towards a great future career.

\section{Central Figure}

A authors' brain-storming session on a conference call.

\section{Article}

Let's make the long story short: life has been completely changed by the COVID-19 pandemic. Since the virus outbreak has mushroomed, people worldwide must accept that, so far, the only possible strategies to "flatten the curve" were lockdown and social distance ${ }^{1,2}$. The need to tailor our life and routine to this new and unique reality was subsequently crystal clear, especially for healthcare professionals (HCPs). At the moment, the oldest and experienced colleagues are facing the urgency to fight against an unknown yet scary disease, while we, as Residents, are forced to deal with both the pandemic and the anticipated delay in our educational programs.

Indeed, if we put the focus on surgery Residents' training, things get further complicated. The best way to improve surgical skills is, of course, practicing in the operating room. Nonetheless, presently trainee's schedule has been disrupted in most of the University Hospitals. In fact, the number of intensive care unit (ICU) beds and ventilation sites has been limited due to their commitment to the COVID-patients' handling. Therefore, the surgical activity has been redirected towards emergent, urgent and not delayable cases. Eventually, due to the in-hospital risk of disease transmission, the number of working doctors has been restricted and some providers have been moved to hospital areas in dire need of physicians experienced in caring for critically ill patients. The post-COVID19 training schedules, as expected, have been switched towards a different model. 
Giving this challenging setting, Surgery Residents assisted to a mandatory shift from regular practice in the operation theatre to "home-sitting" and remote meetings and discussions ${ }^{3}$. For sure, their field of interests had to expand to epidemiological, immunological and pneumological concepts. Whether it is true that the pandemic and the correlated diseases still escape complete comprehension of the mechanisms of spreading and tissue damaging, then every Surgery Resident, who wants to keep up with this new reality, has to dive deep down into the recent broad literature production $\backslash$ sout.

Hence, how could we, on this as Residents, take the pandemic "special" scenario with a grain of salt?

It turns out the daily routine should be assessed out of the OR, trying to get the best from this experience and seizing the opportunity to invest energies and the "extra" leisure on those activities we don't usually have time enough for.

On the basis that we are still "rookie" surgeons, regardless of our individual advance in career, a thorough knowledge of the pathophysiology and the surgical procedures and techniques related to the commonest disorders is crucial. Thus, this seems to be the right circumstance to go back to our desk and study, simply opening the "old but not quite gone" heavy and dusty books, and also to work up our personal skills. Actually, out of this historical crisis could come a moment of enthusiasm, dedication, creativity, innovations and ideas. So, we should take out "pen and paper", or laptops, for the high-tech addicted, and get our thinking caps on: time has come to go deeply in scientific literature, write on a new subject or draw/sketch about anatomy or surgery, to mention just a few, taking benefit likewise from the powerful internet-based libraries.

The SOcial MEdia platforms (SoMe), such as LinkedIn or Twitter, provide an excellent example of the unique opportunity of coupling personal professional advancement with up-to-date technological breakthroughs. HCPs achieve from the SoMe tools to share information, to discuss about health care policy and practice issues, to promote health behaviors, to engage with the public, and to educate and interact with patients, caregivers, students, and colleagues ${ }^{4}$ and provide health information to the community ${ }^{5}$. The role of these networks is undisguised also from the pandemic perspective, since they have been used by world-famous iconic surgeons and doctors to popularize educational daily tips in form of "tweets" or short messages about the disease itself, the way it spreads, the measurements to minimize its diffusion and other related virus-issues.

Beyond chasing a deeper knowledge into our specialty, some of us also chose (or have been forced, given the circumstances) to be personally involved in treating COVID-19 patients. Though at the beginning this appeared just as a different and more selfless way to use our will and time, straightaway it turned out it was a brave leap from our usual training setting. We are facing a crude reality, in which a still elusive disease sustains a lethality rate of $24,6 \%$ in the $70-79$ year age group and $30 \%$ in the $80-89$ one (data from Istituto Superiore di Sanità - Italy). Those bare numbers do not account for the frustration of treating a patient without a validated therapy.

Aside not being academically prepared to face the SARS-CoV-2 pandemic, we perhaps stumbled on our psychological stability: the "usual" setting does not prepare us to such a highly lethal disease, against which we are almost helpless. However, after few days of blue mood, the instinct to survive prevails and teaches us the real meaning of resilience: "get up and try, try" should not be just a chorus, but an everlasting lesson for our career. Beyond professional and logistical thoughts, despite the self-centered universe in which we usually work and live, we found out and realize how deeply human we are today. The sudden solitary confinement we were dragged into ended up to be an optimal starting point to mug the compulsory burnout of our reality. Long duty hours, multiple consecutive shifts and the price of performance are the routine of almost any Cardiac Surgeon and the formed environment of Residents and fellows. Nevertheless, if this step-aside will lead us to find new and creative ways to enhance our learning path, it would have been totally of worth.

To the end, these extraordinary times are an opportunity to demonstrate outstanding collaboration within the Hospital, special staff management, emergency planning, sense of responsibility, innovative ways for Outpatient Care through Telemedicine, virtual meetings and conferences and after all, medical leadership (P.Matt, F,Maisano. Cardiac Surgery and the COVID-19 outbreak: what does it mean? PCR Online 2020). 
Now that we are entering the so called "phase two" the given setting is changing again. First of all, the number of COVID-19 patients is slowly decreasing, as well as the number of dedicated ICU beds. On the other hand, the virus will remain endemic in the society, with an estimated $\mathrm{R}$ value below 1 . As expected, there will be chance to resume the elective surgical procedures, but especially in this moment we need a keen eye on deciding which pathologies have to be treated with priority. For example, Coronary Artery Disease (CAD) showed a higher mortality rate in patients affected by COVID19, but it's however reasonable to think that all the cardiac pathologies affecting the lung circulation - such as symptomatic severe mitral diseases or aortic stenosis - might deserve a priority access to treatment, in order to increase the survival rate in case of an acquired-Coronavirus infection later on.

To the point, The COVID-19 era is teaching us, as doctors and Residents, that we are scientists before anything else. As such, medicine is not meant to be experienced passively, but it should be learnt with everincreasing passion to understand deeply the diseases' mechanisms and the rationale supporting therapies and decisions. As surgeons, even more.

It's plain that we feel the hunger to get a knife in our hands all the time. Anyway, suturing and sewing is not the only way we can improve as physicians and - especially so - our human side. Simply cutting and closing wounds, in a moment in which we are being called upon to show maturity and wisdom, could sound more like a whim. One of the lessons you learn during your surgery fellowship is that our specialty is really multilayered and complex. Awareness of the real meaning behind the mere surgical act of stitching reaches out to gain an insight into our resilient role in this emergency. Getting through the "childish" need and desire to improve our handy skills, we found ourselves far from the leading actors of this pandemic, alone with our desires and ambitions. In that setting the appropriate adult reaction is to develop our future Character, that is the best surgeon we could be.

\section{References}

1. Iacobucci, G. Covid-19: UK lockdown is 'crucial' to saving lives, say doctors and scientists. BMJ 368, m1204 (2020).

2. Lau, H. et al. The positive impact of lockdown in Wuhan on containing the COVID-19 outbreak in China. $J$ Travel Med (2020). doi:10.1093/jtm/taaa037

3. Lewis, E. E., Taylor, L. J., Hermsen, J. L., McCarthy, D. P. \& Fiedler, A. G. Cardiothoracic Education in the Time of COVID-19: How I Teach It. The Annals of Thoracic Surgery (2020). doi:10.1016/j.athoracsur.2020.04.002

4. Bernhardt, J. M., Alber, J. \& Gold, R. S. A social media primer for professionals: digital dos and don'ts. Health Promot Pract 15, 168-172 (2014).

5. George, D. R., Rovniak, L. S. \& Kraschnewski, J. L. Dangers and opportunities for social media in medicine. Clin Obstet Gynecol56, 453-462 (2013). 


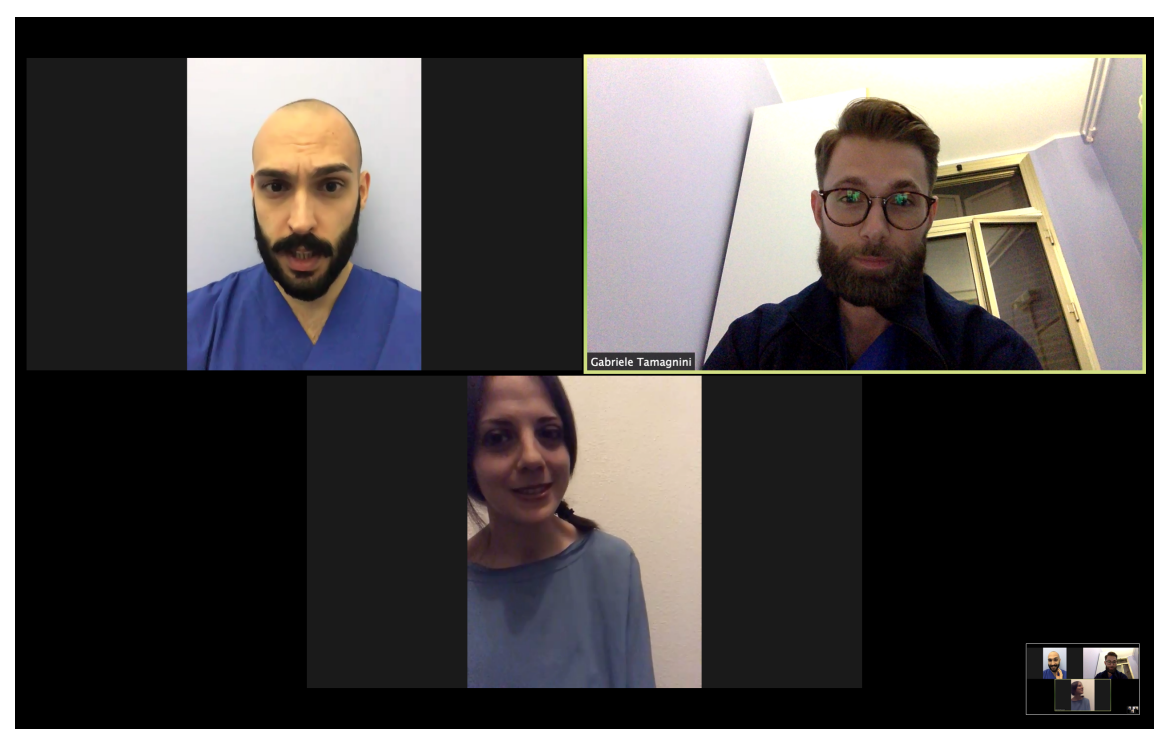

